\title{
Quentius kozeki n. g., n. sp., Nématode rictulaire parasite d'un Marsupial américain
}

\author{
par A.-G. CHABAUD et O. BAIN * \\ * Laboratoire des Vers, associé au C.N.R.S., Muséum national d'Histoire naturelle, \\ 43, rue Cuvier, F 75231 Paris Cedex 05.
}

RESUME. Description de la femelle de Quentius kozeki n. g., n. sp., parasite de Marmosa à Cali (Colombie). A cause de sa structure céphalique, l'espèce est considérée comme le Rictulariidae le plus primitif, actuellement connu, mais elle présente également des caractères aberrants hyperspécialisés.

L'existence de cette espèce semble impliquer que les Rictulaires étaient individualisés dès le Crétacé chez les Marsupiaux américains. Ils se seraient maintenus au moins jusqu'à l'Oligocène chez ces animaux, avant de conquérir les Rongeurs du Nord de la région néarctique, à partir desquels, comme l'a montré Quentin (1971), s'est effectuée une évolution Nord-Sud qui a envahi le monde entier.

Quentius kozeki n. g., n. sp., Rictularid Nematode parasite in an American Marsupial.

SUMMARY. Description of the female of Quentius kozeki n.g., n.sp., parasitic in Marmosa at Cali (Columbia). Because of its cephalic structure, the species is considered to be the most primitive Rictulariidae actually known. In addition, however, it has aberrant and specialized characters.

The existence of this species seems to indicate that the rictularioids were established as early as the Cretaceous in American marsupials. They could have been maintained in these animals at least until the Oligocene before invading Northern rodents of the Nearctic region. From these hosts, as Quentin (1971) demonstrated, there was a North-South evolution throughout the world.

Accepté le 19 juin 1960. 


\section{Introduction}

La région néotropicale située à l'Ouest des Andes est réputée, en zoologie générale, pour sa richesse en espèces reliques. Pour les Nématodes parasites de Vertébrés, le genre Inglamidum Durette-Desset, Diaw et Murua, 1976, découvert au Chili chez un Cricétidé, mais qui est très vraisemblablement issu des Marsupiaux, est le seul Trichostrongyloide néotropical comparable à ceux qui sont parasites des Marsupiaux australiens.

Dans le groupe très différent des Spirurida, la parasitofaune d'un Marsupial de Colombie comporte également une espèce relique remarquable, qui est décrite ci-dessous.

\section{Morphologie}

HôTE :

Marmosa sp. capturé par le $\mathrm{D}^{\mathrm{r}} \mathrm{M}$. Arbarico.

LOCALISATION :

Duodénum : 4 dont l'holotype $-1 / 4$ postérieur intestin-grêle : 1 ?.

LOCALITÉ :

Peñas Blancas, près du Rio Cali, 20 km Sud de Cali. Etat de Valle, Colombie.

Tiers antérieur du corps grêle jusqu'au niveau de la vulve puis très fortement élargi en arrière de celle-ci (fig. 1, A).

\section{- Cuticule :}

Aile cuticulaire dorsale (fig. 1, I) presque aussi large que le diamètre du corps, présente sur le tiers antérieur et disparaissant en arrière de la vulve.

Présence de deux files de 67 épines sur les axes latéro-ventraux du corps. Pointe des épines dirigée en arrière et dorsalement. Il n'y a pas de «peignes » formés par l'imbrication d'épines prévulvaires aplaties comme chez la plupart des autres Rictulaires, mais seulement des épines en "griffe » dont la morphologie est indiquée sur les figures $1, B, L, M, N$. Les deux files d'épines s'écartent de l'axe médio-ventral en arrière de la vulve (fig. $1, \mathrm{~J}$ ), puis se rapprochent de cet axe et s'éloignent à nouveau l'une de l'autre vers les axes latéraux, en arrière du corps. 


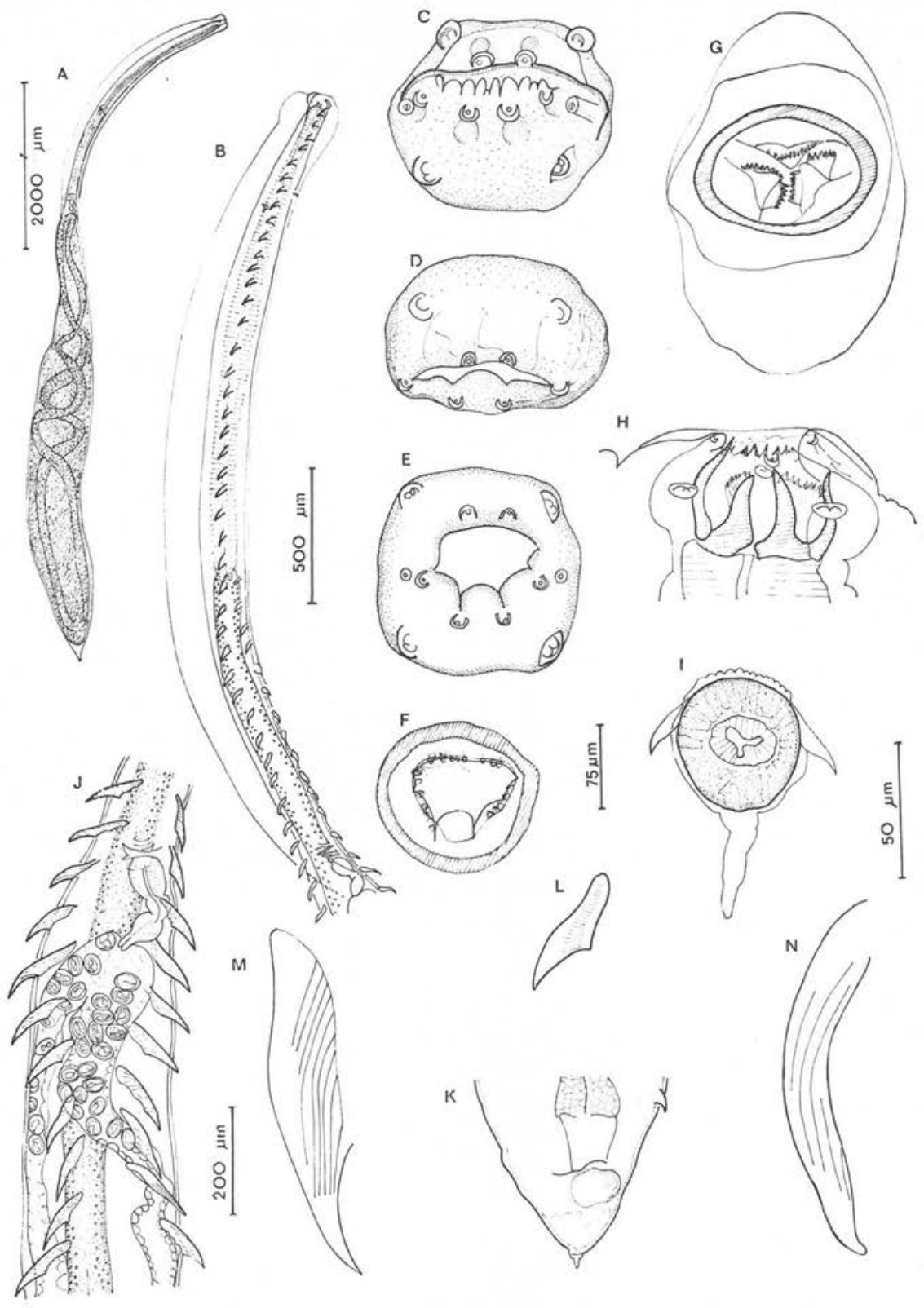

Fig. 1. A : corps entier, vue latérale en avant, ventrale en arrière. B : détail, région prévulvaire. C : tête, vue apicale, bouche fermée. D : id., vue apicale dorsale. E : tête, vue apicale, bouche ouverte. F : id., coupe optique au-dessous de la bouche. G : id., coupe optique profonde. $\mathrm{H}$ : tête, vue latérale droite. I : coupe du corps entre œsophage et vulve. J : région vulvaire, vue ventrale. $\mathrm{K}$ : queue, vue ventrale. $\mathrm{L}: 1^{\mathrm{re}}$ épine. $\mathrm{M}: 37^{\circ}$ épine. $\mathrm{N}: 64^{\mathrm{e}}$ épine. Echelles : A : $2000 \mu \mathrm{m}$; B, K : $500 \mu \mathrm{m}$; C, D, E, F, G, H, K, L, M, N : $50 \mu \mathrm{m}$; I : $75 \mu \mathrm{m}$; 
Anneau nerveux au niveau de la $4^{\mathrm{e}}$ épine, pore excréteur entre $6^{\mathrm{e}}$ et $7^{\mathrm{e}}$ épine, deiride gauche entre $7^{\mathrm{e}}$ et $8^{\mathrm{e}}$ épine, deiride droite au niveau de la $8^{\mathrm{e}}$ épine, fin de l'œsophage au niveau de la $26-27^{\text {e }}$ épine, vulve au niveau de la $39-40^{\circ}$ épine. Les 27 paires postérieures sont de plus en plus largement espacées, le quart postérieur du corps n'ayant plus que 7 paires d'épines.

\section{- TÊTE :}

Structure céphalique proche de celle des Pterygodermatites du sous-genre Paucipectines bien décrite par Quentin (1969) pour $P$. (P.) coloradensis (Hall, 1916), $P$. (P.) microti (Mc Pherson et Tiner, 1942) ou P. (P.) jagerskioldi (Lent et Freitas, 1935).

Les différences sont les suivantes :

1 - La paroi dorsale de la capsule buccale est plus haute que la paroi ventrale. Cependant, les lèvres ventrales plus épaisses surplombent un peu la lèvre dorsale, et la bouche, lorsqu'elle est fermée, n'est pas visible en vue apicale (fig. 1, $C$ et $D$ ). En manipulant le fragment céphalique dans le lacto-phénol entre lame et lamelle, la bouche s'ouvre, et devient bien visible; elle est proche de celle d'un Paucipectines (fig. 1, E).

2 - Les papilles céphaliques et labiales externes tendent à fusionner.

3 - Les trois dents œsophagiennes très puissantes sont ornées, chacune, sur leur face externe, d'une paire de lames verticales dont le sommet est denticulé (fig. $1, G$ et $H$ ). Il y a donc, en arrière de la bordure denticulée sous-jacente à la bouche (fig. $1, F)$, une seconde couronne de denticules, constituée par l'apex des 6 lames cuticulaires insérées sur les 3 dents œsophagiennes.

\section{- CORPS :}

Division entre œsophages musculaire et glandulaire peu marquée. Deirides asymétriques. Vulve très postérieure à l'œsophage s'ouvrant entre 2 lèvres ventrale et dorsale bien marquées. Ovéjecteur figuré en 1, J. EEufs embryonnés occupant toute la moitié postérieure du Vers. Queue figurée en $1, K$, avec tubercule arrondi en arrière de l'anus.

Dimensions de la femelle holotype : longueur du corps $8,7 \mathrm{~mm}$; largeur du corps dans le tiers antérieur $135 \mu \mathrm{m}$, dans le tiers postérieur $640 \mu \mathrm{m}$. Capsule buccale haute de $40 \mu \mathrm{m}$ (dos), à $32 \mu \mathrm{m}$ (ventre) et large de $65 \mu \mathrm{m}$ (axe latéral) à $48 \mu \mathrm{m}$ (axe médian). Aile cuticulaire dorsale haute de $140 \mu \mathrm{m}$. Anneau nerveux, pore excréteur, deiride gauche et deiride droite respectivement à $210 \mu \mathrm{m}, 380 \mu \mathrm{m}, 430 \mu \mathrm{m}$ et $470 \mu \mathrm{m}$ de l'apex. CEsophage long de $1,85 \mathrm{~mm}$. Vulve à $2,9 \mathrm{~mm}$ de l'extrémité antérieure. Cufs de $42 \times 30 \mu \mathrm{m}$. Queue $370 \mu \mathrm{m}$.

MÂlE : inconnu. 


\section{Discussion}

\section{Morphologie.}

Nous considérons le parasite de Marmosa comme le plus primitif des Rictulariidae actuellement connus, car le caractère fourni par l'orientation plus ou moins dorsale de la bouche a une valeur phylogénique essentielle.

Les espèces les plus primitives des Pterygodermatites (rassemblées par Quentin dans le sous-genre Paucipectines) ont une ouverture buccale apicale ou légèrement dorsale, mais la paroi ventrale de la capsule buccale est toujours plus haute que la paroi dorsale, ce qui prépare l'inclinaison de la bouche vers le dos.

Chez le parasite du Marsupial, au contraire, la paroi ventrale de la capsule buccale est plus courte que la dorsale.

L'espèce est également remarquable par sa petite taille et la position reculée de la vulve. Le nombre des paires d'épines (67) est comparable à celui des Paucipectines, mais les épines antérieures sont en "griffes » simples.

Comme chez beaucoup d'espèces reliques, ce parasite a des caractères hyperspécialisés : très grande aile cuticulaire dorsale sur le tiers antérieur du corps, dilatation remarquable de la moitié postérieure du corps.

Nous interprétons donc ce parasite comme une espèce proche-parente des Pterygodermatites les plus primitifs (sous-genre Paucipectines), mais encore plus archaïque que ceux-ci, et ayant en outre des caractères d'hyperspécialisation. Il semble nécessaire de l'isoler dans un genre particulier, Quentius, dédié à notre collègue et ami Jean-Claude Quentin.

\section{DÉFINITION: Quentius n. gen.}

Rictulariidae. Ouverture orale apicale. Paroi ventrale de la capsule buccale moins haute que la dorsale ; 3 dents œsophagiennes armées chacune de deux lames denticulées verticales. Vulve très postérieure à l'œsophage. Epines assez nombreuses et de structure assez uniforme. Grande aile cuticulaire dorsale sur l'avant du corps. Extrémité postérieure du corps enflée. Parasite de Marsupiaux néotropicaux.

Espèce type unique: Quentius kozeki n. sp.

L'espèce est dédiée au $\mathrm{D}^{\mathrm{r}}$ William J. Kozek pour sa généreuse collaboration au cours de notre mission en Colombie.

Un genre encore énigmatique, Rictularoides Hall, 1916, a peut-être quelques rapports avec Quentius. L'espèce type unique $R$. amphiacantha (Diesing, 1851), parasite d'Oxymycterus rufus (Cricetidae) au Brésil, n'a pas été réétudiée depuis sa description originale. La dilatation de l'extrémité postérieure du corps, la présence d'un troisième rang d'épines, pouvant dériver de l'aile cuticulaire dorsale, suggèrent la possibilité, à titre très hypothétique, d'une évolution du genre Quentius dans la région néotropicale. 


\section{Interprétation.}

En se fondant sur l'ontogenèse larvaire et sur une étude morphologique des adultes faite à l'échelle mondiale, Quentin $(1969,1971)$ a individualisé deux lignées distinctes chez les Rictulariidae: le genre Rictularia et le genre Pterygodermatites. Dans les deux cas, les formes les plus primitives se trouvent chez des Rongeurs de la partie septentrionale de la région holarctique et les parasites plus évolués se trouvent chez des animaux variés (Cheiroptères, Insectivores, Primates, Rongeurs, Carnivores) essentiellement en Europe, en Afrique et en Asie.

L'évolution s'est donc faite du Nord au Sud, et sans parallélisme phylétique entre les hôtes et les parasites. En effet, les hôtes des parasites les plus primitifs (Sciuridés, Muroidea) sont apparus tardivement par rapport aux hôtes des parasites les plus évolués (Insectivores, Cheiroptères, etc...). Quentin admet donc une évolution des Rictulariidae relativement tardive, à l'Oligocène, à partir de Nématodes proches du groupe des Seuratum.

L'existence du genre Quentius, plus primitif que les Rictulariidae connus jusqu'à maintenant, mais étroitement apparenté aux Paucipectines, pose donc un problème biogéographique difficile, puisque l'Amérique du Sud est restée insulaire pendant tout le tertiaire. Les Marsupiaux néotropicaux n'ont donc pu avoir aucune connection avec les Rongeurs néarctiques à l'Oligocène.

Par ailleurs, l'évolution morphologique des Rictulariidae et leur répartition géographique ont une ampleur trop grande pour que l'on puisse admettre aisément une évolution quaternaire, postérieure au rétablissement des connections entre Amérique du Nord et Amérique du Sud.

L'hypothèse qui nous semble la plus vraisemblable est donc de supposer que la période d'apparition des Rictulariidae est très antérieure à celle qu'avait suggéré Quentin.

En s'appuyant par exemple sur les données de Jardine et McKenzie (1972), on peut admettre que des ancêtres proches de Quentius - Paucipectines étaient en place dès le Crétacé chez les Marsupiaux américains. Ils ont évolué parallèlement chez ces animaux en Amérique du Sud, où nous retrouvons une faune relique chez Marmosa, et en Amérique du Nord jusqu'à l'Oligocène, début du Miocène, date d'extinction des Marsupiaux.

Les Rictulaires, à ces périodes, ont pu ainsi contaminer les Rongeurs qui étaient en place et entreprendre à partir de cette région l'évolution complexe clairement exposée par Quentin.

\section{Bibliographie}

Jardine N., Mc Kenzie D. P. : Continental drift and the dispersal and evolution of organisms. Nature, $1972,235,20-24$.

Quentin J.-C.: Essai de classification des Nématodes rictulaires. Mem. Mus. Nat. Hist. Nat., nlle sér., sér. A, 1969, 54, 56-115.

Quentin J.C.: Sur les modalités d'évolution chez quelques lignées d'Helminthes de Rongeurs Muroidea. Cah. O.R.S.T.O.M., sér. Ent. méd. Parasitol., 1971, 9, 103-176. 